\title{
RursuSpicae
}

Transmission, réception et réécriture de textes, de I'Antiquité au Moyen Âge

$2 \mid 2019$

Le Physiologus. Manuscrits anciens et tradition médiévale

\section{De proprietatibus quorundam animalium : Le bestiaire du manuscrit 28 d'Avranches}

De Proprietatibus Quorundam Animalium : a Bestiary in the ms. 28 of Avranches Library

\section{Thierry Buquet}

\section{OpenEdition}

\section{Journals}

Édition électronique

URL : http://journals.openedition.org/rursuspicae/540

DOI : $10.4000 /$ rursuspicae. 540

ISSN : 2557-8839

Éditeur :

Université Nice-Sophia Antipolis, IRHT - Institut de recherche et d'histoire des textes

\section{Référence électronique}

Thierry Buquet, «De proprietatibus quorundam animalium : Le bestiaire du manuscrit 28 d'Avranches », RursuSpicae [En ligne], 2 | 2019, mis en ligne le 20 décembre 2019, consulté le 29 mars 2021. URL: http://journals.openedition.org/rursuspicae/540 ; DOI : https://doi.org/10.4000/rursuspicae.540

Ce document a été généré automatiquement le 29 mars 2021.

RursuSpicae 


\title{
De proprietatibus quorundam animalium : Le bestiaire du manuscrit 28 d'Avranches
}

\author{
De Proprietatibus Quorundam Animalium : a Bestiary in the ms. 28 of \\ Avranches Library
}

Thierry Buquet

1 Lors du catalogage des manuscrits conservés à Avranches, réalisé dans le cadre de la Bibliothèque virtuelle du Mont Saint-Michel (BVMSM), j'ai été amené à m'intéresser à un court texte conservé dans un manuscrit composite portant la cote $28^{1}$. Le texte est un Physiologus, seul traité de ce type conservé à Avranches qui soit issu du fonds du Mont Saint-Michel. Peu de bestiaires subsistent dans les bibliothèques normandes, et quelques-uns seulement, enluminés ou non, ont été produits dans les scriptoria normands ${ }^{2}$. La présente contribution a pour modeste objectif de signaler une version inédite du Physiologus empruntant, comme nous le verrons, aux versions A, B et $Y$ et un nouveau témoin manuscrit à la complexe tradition manuscrite et philologique des bestiaires latins ${ }^{3}$.

\section{Le manuscrit 28 d'Avranches}

Le codex 28 d'Avranches, en parchemin, in-quarto $(240 \times 160 \mathrm{~mm}), 232 \mathrm{ff}$., est le fruit du regroupement d'au moins deux manuscrits - cotes anciennes Le Michel (SS4 et S10 aux f. 1 et f. 122$)^{4}$, reliés ensemble par les Mauristes lors de la restauration des reliures au $\mathrm{XVII}^{\mathrm{e}}$ siècle. Il n'est pas enluminé, à l'exception de belles initiales ornées dans la première unité. La reliure, typique de la période mauriste, réalisée en ais de carton sur 4 nerfs doubles, est en mauvais état et le parchemin est de conservation très variable selon les unités codicologiques. L'ais inférieur, couvert de peau brune, porte un décor de triple filet à froid. L'ais supérieur a été remplacé par du carton moderne ( $\mathrm{xIX}^{\mathrm{e}} \mathrm{s}$.) et renforcé par du papier de la même époque. La garde inférieure est en papier, du même 
type que celui utilisé lors des restaurations mauristes. Il ne s'agit pas d'un codex d'apparat, mais d'un manuscrit de travail.

3 D'après Colette Jeudy ${ }^{5}$, la première partie, datant $\mathrm{du}^{\mathrm{XII}} \mathrm{e}^{\mathrm{e}}$ siècle, est originaire du nordouest de la France, probablement de l'abbaye du Mont Saint-Michel. Cela semble moins certain pour les autres parties, datables du XIII ${ }^{\mathrm{e}}$ siècle. Léopold Delisle, dans le Catalogue général des manuscrits, affirmait que l'ensemble $\mathrm{du}$ manuscrit était «vraisemblablement» originaire du Mont ${ }^{6}$. Il n'y a aucun ex-libris ou marque de possession dans le manuscrit. Identifier son origine semble quelque peu aléatoire, car il pourrait s'agir, pour les parties datables du XIII ${ }^{e}$ siècle, d'un manuscrit offert au Mont ou acquis par l'abbaye, par exemple auprès d'un maître parisien ${ }^{7}$, sans qu'aucun élément factuel ne vienne confirmer ou infirmer cette possibilité.

4 Le volume contient 9 unités codicologiques : 4 dans le premier manuscrit ; 5 dans le second. Les cahiers sont en majorité des quaternions (23 cahiers sur 33$)^{8}$. Le premier manuscrit (cote Le Michel SS 4, ff. 1-121v) contient des varias bibliques: les Épitres de Paul (f. 1r-58v), correspondant à la première unité codicologique, à longues lignes, du $\mathrm{XII}^{\mathrm{e}}$ siècle. La réglure est à la pointe sèche uniquement dans cette partie du manuscrit. Par la suite, on observe la présence irrégulière de réglures à l'encre. Dans les parties suivantes, à partir du f. 59r, la mise en page se présente en deux colonnes, avec une écriture du XIII ${ }^{e}$ siècle. Mais il existe plusieurs types de réglure, avec des justifications de largeurs variables. Ces variations codicologiques importantes indiquent que le manuscrit anciennement coté Le Michel SS 4 était lui-même le fruit d'un regroupement de deux volumes distincts. La $2^{\mathrm{e}}$ unité codicologique (59r-92v) contient des gloses (dont un fragment de celle de Pierre Lombard sur les Psaumes ${ }^{9}$ ), des commentaires bibliques et des traités moraux (sur les anges, sur les vices et vertus), ainsi qu'un extrait de Martin de Braga (Formula honestae vitae. Ad Mironem regem Galliciae I-III) ${ }^{10}$. C'est un manuscrit peu soigné : il s'agit sans doute de notes de travail. Des courts textes sur des bandes de parchemin de petit format ont été insérées au moment de la reliure : un bref traité intitulé De angelis, écrit sur le recto d'un feuillet (f. $69 \mathrm{v}$ et $78 \mathrm{r}$ ), le verso est vierge. Le feuillet est constitué de deux petites pièces de parchemin cousues ensemble (voir couture f. 69v).

$5 \quad$ La $3^{\mathrm{e}}$ unité codicologique (ff. 93r-120r) comprend un long commentaire non identifié sur l'Épitre de Paul aux Corinthiens (Expositio in epistolam Pauli ad Corinthios). La mise en page et la copie sont plus soignées, en deux colonnes régulières. La $4^{\mathrm{e}}$ unité (ff. 121r-122v) présente quelques commentaires bibliques anonymes et des extraits des Disticha catonis, relevés par Colette Jeudy dans son catalogue des classiques latins ${ }^{11}$.

6 La suite du codex correspond à la cote Le Michel S10 (ff. 123-232v). C'est dans cette partie la plus complexe que se trouve le bestiaire. Dans le Catalogue général des manuscrits, Delisle le décrit comme un «mélange confus de brouillons sur toutes sortes de sujets; des lambeaux d'ouvrages (...) Ce sont probablement des matériaux à l'usage des prédicateurs $»^{12}$. Le Michel lui donne pour titre Sermones farrago inordinata (compilation désordonnée de sermons) ${ }^{13}$.

7 Ce second manuscrit est le fruit de la réunion de cahiers divers; le parchemin est de moins bonne qualité, les feuillets y sont de tailles variables. L'écriture est de très petit module, dans un latin très abrégé. De l'encre rouge a été utilisée pour surligner des rubriques et colorer les pieds de mouches. On note également la présence de nombreux schémas arborescents, déclinant entre autres des ensembles de vices et de vertus. Dans 
la liste suivante, les unités codicologiques, manquantes, de 1 à 4 , correspondent à la première partie de manuscrit datant du XII ${ }^{\mathrm{e}}$ siècle.

- $5^{\mathrm{e}}$ (f. 123-138v) : Traité sur messe de Noël ; gloses bibliques ;

- $6^{\mathrm{e}}$ (f. 139-195) : Partie complexe qui contient le bestiaire (détaillée ci-après)

- $7^{\text {e }}$ (f. 196-207v) : Sententiae, gloses et interprétations de la Bible et de divers auteurs ;

- $8^{\mathrm{e}}$ (f. 208-227) : Glose anonyme sur le livre des Juges ;

- $9^{e}$ (f. 228-232) : Notes théologiques diverses, recettes médicinales et distiques d'Hugo Prima.

8 La $6^{\mathrm{e}}$ unité (f. 13r-195r), composée de 6 cahiers (19 à 26, voir note 8), contient un ensemble de textes religieux pour la prédication : des commentaires sur les Évangiles, un traité sur les vertus (signalés par des rubriques), des sermons ${ }^{14}$, un bestiaire qui commence avec un nouveau cahier (le $\mathrm{n}^{\circ} 25$ ), un recueil d'exempla, un second traité sur les vices et vertus (notamment sur l'espérance, la joie, etc.) et des Sermones de Demoniaco.

\section{Du bestiaire...}

Le texte du bestiaire court sur seulement deux feuillets en double colonne, d'une écriture de petit module (ff. 179r-180r). Chaque chapitre est signalé par une rubrique surlignée en rouge; l'initiale de chaque incipit est surlignée de même. Le texte est rédigé dans un latin très abrégé ; il s'agit d'une copie rapide non exempte de fautes et de lacunes textuelles. Le traité est composé de 30 chapitres (pour 31 notices). Le titre du texte est rubriqué à l'encre rouge: De proprietatibus quorundam animalium «Propriétés de quelques animaux». Le mot proprietates évoque les encyclopédies du xIII siècle, comme le De proprietatibus rerum de Barthélemy l'Anglais, mais surtout les recueils de propriétés naturelles, qui servaient directement aux prédicateurs pour l'écriture des sermons. Je n'ai pas trouvé d'intitulé équivalent dans les bestiaires, pour ceux qui portent un titre, où l'on fait plutôt mention de la «nature » des bêtes : Liber Physiologus de natura animalium vel avium seu bestiarum (Oxford, Bodleian, Auct. T.2.23, f. 127, témoin du Physiologus B) ; Bestiarum loquens de naturis omnium bestiarum (Paris, Bnf Lat. 6838B), Liber de natura bestiarum (British Library, Harley MS 3244, bestiaire de la $2^{\mathrm{e}}$ famille), Liber de naturis bestiarum et earum significationibus (British Library, Royal MS 12 C XIX, famille "Transitional»), Liber de naturis Bestiarum ${ }^{15}$, De natura quorundam animalium (Rouen BM, ms 1468, $2^{\text {e }}$ famille) ${ }^{16}$, etc. Le terme de proprietas est absent non seulement des titres de bestiaires, mais aussi des textes mêmes, au bénéfice de natura, que l'on trouve à la fois dans les titres, comme nous venons de le voir, mais aussi dans les chapitres consacrés aux animaux; ainsi le lion a trois « natures" (Physicii dicunt leonem tres principales natures habere $\left.{ }^{17}\right)$. Dans le bestiaire d'Avranches, natura apparaît douze fois, et proprietas jamais.

10 Si l'on peut d'une part considérer natura comme le caractère originel, l'essence d'une chose et d'autre part proprietas comme le caractère particulier ou spécifique ${ }^{18}$, il n'est pas évident que cette distinction soit toujours bien claire pour un lettré du XIII siècle. Thomas de Cantimpré, dans son encyclopédie intitulée Liber de natura rerum, explique notamment que les propriétés sont multiples, l'essence d'une chose est unique (proprietates quidem diverse sunt, sed una essentia; Eadem est essentia, sed proprietas divers $a^{19}$ ); dans son prologue il différencie bien natura de proprietas: que de naturis rerum, creaturarum et earum proprietatibus ${ }^{20}$. Pour Thomas, proprietas désigne une caractéristique propre, parmi d'autres ; de reste ce terme est souvent utilisé au pluriel 
(comme chez Barthélemy l'Anglais), chaque objet ou être vivant pouvant avoir plusieurs « propriétés » ${ }^{21}$.

11 Mais dans le cas du bestiaire d'Avranches, ne peut-on pas considérer que l'auteur donne le même sens à nature et propriété ? Peut-être a-t-il substitué un terme fréquemment utilisé au XIII ${ }^{\mathrm{e}}$ siècle, proprietas, notamment dans les traités d'histoire naturelle, à celui de natura, hérité des anciennes versions du Physiologus. Malgré son titre, le bestiaire du ms. 28 ne peut être rapproché du genre littéraire des listes ou recueils de propriétés, étudié par Emmanuelle Kuhry, où les animaux et autres réalités naturelles sont classés alphabétiquement, chaque chapitre donnant une liste de propriétés synthétisées en quelques mots, souvent sous le mode étymologique, comme dans le recueil intitulé Angelus purus natura. Par contre, l'utilisation du terme proprietas signifie sans doute un usage du texte dans le cadre de la prédication ${ }^{22}$ : la connaissance des "propriétés des choses » était utilisée comme moyen de penser le monde naturel, dans un objectif d'étude de la nature, et pour la prédication ${ }^{23}$. Thomas de Chobham, dans sa Summa de arte praedicandi, explique que le prédicateur doit connaître les natures des animaux et des choses (naturas animalium et aliarum rerum) parce que rien ne peut plus émouvoir le cœur de l'auditoire que les propriétés des animaux (proprietas animalium). Dans ce passage, l'auteur ne semble pas faire de différence nette entre natura et proprietas, termes qu'il utilise d'ailleurs au pluriel. Il ajoute aussi que les analogies avec les choses (similitudines rerum) transportent l'âme plus facilement et de façon plus agréable ${ }^{24}$.

\section{...au recueil d'exempla et de similitudines}

12 Avant de présenter le contenu du bestiaire en détail, il faut signaler qu'il existe une rupture textuelle au f. 180r, où un discret pied de mouche introduit un autre texte, dont le titre est donné par la rubrique suivante : Ecce similitudines multe de diversis et primo de carnifice. Le terme de similitudines indique que nous sommes toujours dans un contexte d'exégèse allégorique. Ce texte se poursuit jusqu'au f. 181r. Avant ce pied de mouche, les deux chapitres finals du bestiaire (de apicula et un sans titre qui parle des oiseaux) sont en fait des exempla et non des paragraphes inspirés du Physiologus. Le compilateur semble s'être rendu compte qu'il était passé du domaine des bestiaires à celui des exempla, et a alors ressenti le besoin de marquer en cet endroit une séparation. Néanmoins, la mise en page reste la même : chaque court chapitre est signalé par un pied de mouche, et une rubrique rehaussée de couleur rouge. La transition entre bestiaire et recueil de similitudines n'est donc pas nette. De plus, l'avant-dernier paragraphe du f.181r concerne les pierres ardentes: il s'agit d'un passage repris fidèlement du Physiologus, dans sa version $B^{25}$. C'est également le dernier chapitre du Bestiaire de la seconde famille, dans une version très proche ${ }^{26}$. L'auteur revient ici au bestiaire, avec un chapitre qui aurait pu se trouver dans la partie intitulée $D e$ proprietatibus quorundam animalium. Le recueil Ecce similitudines multe de diversis constitue un recueil de "similitudes" et d'exempla, dont certains sont relatifs aux animaux : cheval, crapaud, âne, poisson, corbeau, chien, porc, autour, loup, mouton, chien. On décompte 64 exempla, dont 16 mettent en scène des animaux (soit un quart). Entre les ff. 179r et 181r, le manuscrit présente à la fois des discontinuités multiples, mais conserve une même apparence, en traitant des sujets naturalistes où l'animal a une place privilégiée dans un cadre de moralisation allégorique de la nature. Cela nous 
renvoie à ce que nous évoquions plus haut chez Thomas de Chobham, où proprietas et similitudo peuvent avoir chacun un rôle important dans la prédication ${ }^{27}$.

Concernant la partie relative aux exempla et similitudines, une rapide recherche sur le Thesaurus exemplorum medii aevi (ThEMA) ${ }^{28}$ a permis de voir que certains exempla semblent assez courants car on les trouve chez les compilateurs des XIII ${ }^{\mathrm{e}}$ et XIV ${ }^{\mathrm{e}}$ siècles. J'en donne ici quelques exemples, dont je traduis et résume sommairement la teneur, et signale les parallèles chez d'autres auteurs :

- Nature $d u$ corbeau. Le corbeau ne reconnaît pas ses petits jusqu'à ce que leurs plumes noircissent et lui ressemblent. Ainsi, Dieu ne nourrit pas les hommes tant qu'ils ne lui ressemblent pas en vertu (parallèles constatés dans les Sermones aurei de Jacques de Voragine et chez Étienne de Bourbon) ${ }^{29}$.

- Ruse du loup. Le loup épargne la brebis tant qu'il ne se trouve pas dans un endroit sûr et isolé, de même le diable ne s'attaque pas au pêcheur, tant qu'il est sous l'influence du prédicateur (parallèle dans le ms. London, British Library, Add. 27909 B, De seducione diabolica) ${ }^{30}$.

- Le chien qui suit deux personnes finira toujours par suivre son maitre (parallèles chez Odo de Ceritona, dans le Ci nous dit ${ }^{31}$ et dans le ms. London, B.L., Add. 27909 B²).

- Autre exempla sur le chien. Un chien porte dans la gueule un fromage qu'il a dû dérober à quelque fenêtre, ou dans une maison. Passant sur un pont, il voit l'ombre du fromage dans l'eau, croit que c'est là son fromage ou un autre, ouvre la gueule, saute dans l'eau, perd son fromage et se noie (parallèle chez Ranulphe de la Houblonnière ${ }^{33}$ ).

- Histoire de l'insensé qui dort dans la fange et couche son bétail sur de beaux tapis (London, B.L., Add. 27909 B et Ci nous dit ${ }^{34}$ )

14 J'ai repéré une autre source, en dehors de ThEMA : l'exemple d'un poisson pris dans la nasse qui ne peut en sortir; il est alors comparé au pêcheur prisonnier de ses vices. Cette comparaison peut se trouver chez de nombreux auteurs, mais en général à propos de la gourmandise des poissons (un sermon de Guillaume d'Auvergne ${ }^{35}$, dans le Liber de exemplis ac similitudinibus rerum de Giovanni da Sancto Geminiano ${ }^{36}$, ou encore chez Bernardin de Sienne ${ }^{37}$ ).

Plusieurs rubriques indiquent que certaines notices sont des exempla: De peregrino exemplum; aliud exemplum; de même, dans le texte, on trouve plusieurs mentions de type hoc exemplum, etc., mais dans ces cas l'auteur appelle exempla ce qui pourrait être plutôt qualifié de similitudines, des comparaisons allégoriques. Par exemple, sous la rubrique "aliud exemplum », le texte contient deux fois le mot similiter. Ces confusions entre exempla et similitudines sont assez fréquentes au Moyen Âge, car elles sont très proches dans leur forme et leur contenu ${ }^{38}$.

\section{Structure et sources du Physiologus d'Avranches}

L'objectif de cette partie est de parvenir à identifier les sources du bestiaire, et d'éventuellement de la classer dans la complexe tradition textuelle du Physiologus et de ses dérivés. Je me suis d'abord intéressé à l'ordre des chapitres tels qu'ils se présentent dans le ms. 28 : en effet, c'est un bon moyen de le comparer à d'autres versions, qui présentent un classement et un nombre de notices très variables. Dans le même ordre d'idée, les animaux mentionnés ou absents donnent un bon élément de comparaison avec les autres familles de textes. J'ai également questionné l'utilisation du marqueur 
de citation de citation Physiologus, pour essayer d'y trouver un indice supplémentaire d'utilisation de sources particulières.

\section{L'ordre des chapitres}

17 Les 31 notices du bestiaire d'Avranches ne se présentent pas dans un ordre semblable à celui des diverses versions du Physiologus. Dans l'immense majorité des cas, c'est par le lion que le texte commence. Dans le ms. 28, il n'est placé qu'en douzième position, et ce chapitre commence par l'incipit habituel du Physiologus B, et de ses dérivés directs: Etenim Iacob, benedicens filium suum Iudam, ait: Catulus leonis Judas, filius de germine meo, quis suscitabiteum ? ${ }^{39}$.

C'est la licorne (unicornis) qui ouvre le texte d'Avranches, alors qu'elle ne se trouve pas en tête des bestiaires "canoniques ». Deux témoins manuscrits des Dicta chrysostomi (München BSB Clm 5613 et $\mathrm{Clm}$ 14216) font cependant exception ${ }^{40}$, mais la notice sur la licorne du ms. 28 ne s'en inspire pas ${ }^{41}$. Elle ne présente qu'un lointain rapport avec le Physiologus $\mathrm{B}$, dont elle ne garde que quelques éléments descriptifs très abrégés de la notice, et dont les interprétations allégoriques sont très différentes.

L'ordre des chapitres observé dans le ms. 28 ne correspond à aucune version recensée du Physiologus; néanmoins, l'ensemble présente de nombreux parallèles avec la version $\mathrm{B}^{42}$, dont est copiée intégralement une dizaine de chapitres dans le seconde moitié du texte. D'autres notices, dans la première partie du bestiaire, présentent certaines similarités avec B, duquel Avranches ne garde que les propriétés naturelles, mais ne conserve pas les interprétations allégoriques, comme dans la séquence cervus-erinaciusvulpis (voir annexe 1).

Dans la liste des chapitres, quelques séquences présentent le même ordre que la version B : castor, hyène, caprea, onagre. Le chapitre hydrus, présenté en détail plus loin, vient juste après, alors qu'il aurait dû se retrouver avant caprea, selon l'ordre du Physiologus B. On retrouve cette séquence dans les versions directement issues de B, notamment BIsidore : mais aucun rapprochement n'est possible avec cette dernière version, car le manuscrit d'Avranches ne donne aucune considération étymologique. Il faut néanmoins évoquer une autre possibilité : le compilateur a aussi pu laisser de côté les étymologies qui sont clairement signalées dans la plupart des témoins de B-Isidore, et toujours situées en fin de notice ${ }^{43}$.

D'autres chapitres, placés dans la première moitié du bestiaire, sont empruntés, au moins pour partie, à la version $Y$ (hirundo, herodius, cervus, perdix, mustela). La notice sur l'oiseau herodius dépend uniquement de $\mathrm{Y}^{44}$. Cet oiseau est présent dans le Physiologus B sous la rubrique fulica (terme donné comme synonyme à herodius dans le Physiologus Y), mais la notice de B est sans rapport avec celle du ms. 28.

Le bestiaire d'Avranches est donc composé de deux parties, aux sources différentes :

1. jusqu'au chapitre 11 (mustela), c'est une partie peu dépendante de B, influencée partiellement par $\mathrm{Y}$, qui présente des moralisations originales par rapport à $\mathrm{B}$ et à $\mathrm{Y}$ et qui contient un chapitre ne provenant ni de B ni d'Y (milvus) ;

2. à partir chapitre 12 (leo), le texte est proche de $\mathrm{B}$, les notices 21 à 28 étant strictement copiées sur cette version. 


$$
\begin{aligned}
& \text { personnelle, avant de se conformer ensuite à une compilation plus stricte d'un témoin } \\
& \text { ou d'un transmetteur de B. }
\end{aligned}
$$

\section{Le marqueur de citation Physiologus et la version B}

Le compilateur a gardé ou inséré des marqueurs de citation «Physiologus» (de type Physiologus describit, dicit Physiologus, etc.) : ils sont présents dans 9 notices sur les 31 . Ces références se trouvent principalement dans la séquence des chapitres 20-28, lesquels sont très proches du Physiologus $\mathrm{B}$, et dans la notice sur l'oiseau caladrius, elle aussi proche de $B$ (voir annexe 1 ). Ces marqueurs de citations sont très présents dans la version B : 27 chapitres sur les 37 en présentent un au minimum. Parmi les 25 entrées zoologiques communes à Avranches et au Physiologus B, 4 n'ont pas de marqueur de citation dans B : simia, nycticorax, serra et antelops, absence que l'on observe aussi dans le manuscrit d'Avranches. Dans la séquence des chapitres 20-28 d'Avranches, une seule notice ne présente pas de marqueur Physiologus, celle du singe (simia), comme dans son modèle, le Physiologus B. Outre la ressemblance générale entre les notices, l'utilisation commune à son modèle de marqueur de citation « Physiologus dicit... ", confirme la copie d'après la version $B$.

Faut-il pour autant en conclure que les autres chapitres, qui n'ont pas de marqueur de citation, et qui présentent des différences avec B, ont une autre source que B ? Tous les chapitres sans marqueur Physiologus (cf. tableau, annexe 1) sont relativement éloignés de $B$ (sauf pour le lion et l'ibis, très proches de $B$ ). On constate cependant que dans ces chapitres le copiste a tendance à ne reprendre que les principales propriétés sur les animaux, en les résumant. Il se peut qu'en résumant son propos, il ait fait aussi disparaître le marqueur Physiologus.

La notice de l'oiseau caladrius est littéralement proche de celle du Physiologus B. La phrase commençant par Sicut leo et aquila... résout la contradiction apparente entre d'une part le fait que le lion et l'aigle sont impurs dans la loi biblique, et d'autre part qu'ils sont pourtant assimilés au Christ, les deux animaux étant respectivement roi des animaux et roi des oiseaux ${ }^{45}$. La phrase commençant par Et sic multa sunt in creaturis... ${ }^{46}$ est un écho de la fin de la notice de la version $\mathrm{Y}^{47}$ et un décalque exact de la phrase qu'on trouve dans la version $\mathrm{B}^{48}$. Cette phrase est relative à la double interprétation possible de certaines créatures, en bonne ou en mauvaise part, selon le contexte.

\section{Les animaux non mentionnés dans le bestiaire d'Avranches}

Le Physiologus B compte au total 36 chapitres, pour 31 dans le ms. 28. Cinq animaux du ms. 28 n'existent pas dans B, mais on constate également l'absence de plusieurs animaux: éléphant, autruche (asida), panthère, colombe, fourmi, aspidochelone (baleine $)^{49}$. Ces manques sont surprenants car éléphant, colombe, panthère et autruche peuvent être considérés comme des vedettes des bestiaires. Il faut noter que l'éléphant et la colombe sont absents du manuscrit de référence de l'édition de la version B par Carmody, nommé d'après le manuscrit de Bern, Bürgerbibl., Lat. 233. Panthera, aspidochelone, asida, elephas et columba sont placés en fin de la version B. L'édition de Carmody a ajouté la séquence columba, peredixion, elephans, amos, adamas, margarita, lacerta d'après d'autres versions du Physiologus ${ }^{50}$ tirées entre autres ${ }^{51}$ de témoins de la 
version B-Isidore ${ }^{52}$, alors que le manuscrit de Bern ne compte que 32 chapitres. Par exemple, dans le chapitre sur la colombe, Carmody s'appuie sur 9 manuscrits, mais pas sur Bern, Lat. 233, ni sur Oxford, Bodleian Libr., Auct. T.2.23, les seuls témoins de B connus à l'époque de Carmody, qui ne comportent pas la séquence finale de columba à margarita. Seul le manuscrit d'Oxford mentionne ces chapitres dans sa table des matières, ce qui explique les ajouts opérés par Carmody. Il faut aujourd'hui signaler le manuscrit Montecassino, Archivio dell'Abbazia, 323, du IX siècle, qui comporte bien les chapitres perdus dans les manuscrits de Bern et d'Oxford, et qui explique donc à la fois la table des chapitres du manuscrit d'Oxford et la structure de la version B-Isidore ${ }^{53}$.

La séquence finale du manuscrit de Bern est la suivante : panthera, aspichelone, perdix, mustela, aspis, asida, turtur, cervus, salamandra ${ }^{54}$. Perdix, herodius, mustela, turtur, cervus, salamandra (stellio) sont présents dans Avranches. Seul stellio est proche de B (chapitre salamandra), dans une forme abrégée, qui reprend la première moitié de $B$, avec dans les deux cas un marqueur Physiologus, mais résume très fortement la seconde partie, en raccourcissant notamment les citations bibliques. Les autres textes sont plus éloignés de la version B. Par exemple, la notice du cerf du ms. 28 est beaucoup plus réduite que celle de B et elle ne contient pas de marqueur Physiologus. Il faut signaler également que dans le ms Oxford BL, Auct. T.2.23, ff. 127-155 (Ixe siècle), on ne trouve ni éléphant ni colombe - la table des chapitres donne 37 entrées, mais le traité se termine par « Finit » après le $31^{\mathrm{e}}$ chapitre (salamandre), à l'instar du manuscrit de Bern qui compte lui aussi 31 chapitres $^{55}$.

Le compilateur du manuscrit d'Avranches a-t-il eu accès à un témoin manuscrit incomplet de $\mathrm{B}$, dans lequel les derniers chapitres auraient été absents, ou bien à un manuscrit dérivé de celui de Bern, ne contenant qu'un nombre réduit de chapitres? C'est une explication probable justifiant l'absence de l'éléphant de la colombe et des autres. Néanmoins, on ne peut écarter totalement l'hypothèse qu'Avranches ne soit qu'une copie partielle d'un modèle plus complet.

\section{Comment situer le Physiologus d'Avranches dans la famille des bestiaires?}

Le Physiologus d'Avranches ne présente aucun point commun avec les bestiaires des seconde et troisième familles, où le nombre et la variété des espèces décrites est beaucoup plus important; il n'est pas non plus inspiré de la version B-Isidore, le texte d'Avranches ne donnant aucun élément étymologique. Les seuls points communs sont dus à la proximité avec $\mathrm{B}$, qui est la base de toutes les versions ultérieures. La source principale du Physiologus d'Avranches est B et c'est manifeste pour la seconde moitié des entrées. Mais le scribe a peut-être utilisé une autre version, parfois contaminée par $\mathrm{Y}$, comme nous l'avons vu plus haut; la version A pourrait être une autre bonne candidate ${ }^{56}$, à ceci près que certains chapitres non tirés de $B$, comme turtur, n'ont aucun rapport avec $\mathrm{A}^{57}$. Cette «copie de $\mathrm{B}$ » est également identifiable, comme nous l'avons déjà évoqué, par la présence de marqueurs de citation Physiologus, dans les séquences de chapitres directement tirées de B; la compilation souvent scrupuleuse du contenu des notices du Physiologus B ; enfin, dans cette partie compilatoire, il a probablement utilisé une copie incomplète de $B$, dans une tradition manuscrite perdue issue $d u$ manuscrit de Bern ${ }^{58}$. Il existe dans un manuscrit des XII ${ }^{\mathrm{e}}$-XIII ${ }^{\mathrm{e}}$ siècle, Rouen, B.M. 638, un Physiologus (De naturis animalium, ff. 232-239v) ${ }^{59}$, dans une version très proche de $\mathrm{A}$, qui 
présente les mêmes lacunes que le bestiaire d'Avranches: les chapitres éléphant, autruche, panthère, aspidochelone y sont également absents. Presque tous ces chapitres se trouvent en fin du Physiologus B, comme nous l'avons vu. Avranches et Rouen présentent des séquences dans un ordre identique (comme par exemple la série Onager - Hydrus - Simia dans cet ordre), notamment des chapitres qui sont dans les deux cas très proches du Physiologus B. Les deux manuscrits ont-ils pu procéder d'un modèle commun? L'hypothèse est séduisante, d'autant que le manuscrit de Rouen provient de l'abbaye Saint-Ouen de Rouen. Si l'on considère de façon hypothétique que l'origine du manuscrit d'Avranches est normande, il aurait pu circuler en Normandie un modèle commun inspiré de B et proche de A. Mais de nombreux éléments viennent affaiblir cette hypothèse: certains chapitres de Rouen sont absents d'Avranches (fourmi, monoceros, Ysida/struthiocamelus "autruche», colombe); Rouen n'a pas certaines notices d'Avranches (hirundo, milvus, cervus, Ybis, mustela, etc.). Ensuite, d'autres chapitres présentent des textes assez divergents : lion, antilope, aigle, renard, castor, perdrix, tourterelle. Si l'on compare avec le manuscrit de Bruxelles, KBR 10074, qui présente une version plus complète que Rouen de la version A, les notices hupupa, herodius et herinacius (absents de Rouen) présentent de très fortes variations avec les chapitres correspondants dans Avranches. Les rapports entre la version d'Avranches et le Physiologus $\mathrm{A}$ sont donc très ténus et s'inscrivent surtout dans une proximité avec $\mathrm{B}$, à la fois dans le contenu textuel et dans l'ordre de certains chapitres.

31 Dans la première partie, le rapport aux différentes versions du Physiologus est moins évident, malgré des points communs avec Y. Les oiseaux hirundo et milvus sont absents du Physiologus B. Milvus ${ }^{60}$ est très rare dans les bestiaires, et seulement présent dans certains manuscrits des seconde ${ }^{61}$ et troisième familles. On le trouve dans l'Aviarum d'Hugues de Fouilloy, mais le texte, qui s'inspire d'Isidore de Séville, n'a rien en commun avec celui $d^{\prime} A^{\prime}$ ranches $^{62}$, où la source est biblique: le milan aurait une connaissance innée du décompte du temps ${ }^{63}$; cette propriété ne se trouve pas chez Pline, ni chez Isidore, ni dans les différentes versions du Physiologus. Pour confirmer cette hypothèse, il faut mentionner ici les encyclopédistes Thomas de Cantimpré ${ }^{64}$ et Vincent de Beauvais ${ }^{65}$, qui citent cette propriété du milan uniquement d'après la Bible et la Glose ordinaire, avec la même citation que celle utilisée par le compilateur du manuscrit 28. Il est bien établi que la Bible et la Glose ordinaire sont des sources d'histoire naturelle essentielles pour les compilateurs et les encyclopédistes médiévaux ${ }^{66}$. Dans la première partie du bestiaire, le scribe du manuscrit 28 n'utilise pas le marqueur de citation Physiologus, et semble s'inspirer d'abord de citations bibliques (par exemple pour les chapitres unicornus, turtur, hirundo). Il travaille également sur un fonds commun de propriétés qu'on trouve dans plusieurs versions les bestiaires (notamment $\mathrm{B}$ ), propriétés qu'il résume et abrège, en créant souvent des moralisations et comparaisons allégoriques originales, qu'il intrique avec les éléments descriptifs, réorganisant ainsi les notices.

32 Si l'inspiration principale du compilateur est bien le Physiologus B, il s'agit donc d'une copie très "contaminée ", ce qui n'est pas si rare dans la complexe tradition manuscrite des bestiaires ${ }^{67}$. Cette "contamination» s'exprime par un usage de la compilation abrégée du Physiologus, par l'apport d'autres sources (par exemple, la Bible glosée), par des emprunts à $Y$ et par des interventions directes plus personnelles dans les moralisations, où, par exemple, il créé des mentions à l'impiété et aux péchés des juifs dans les chapitres milvus et turtur. Pour ce dernier, il ajoute une comparaison 
inédite entre cet oiseau et la Vierge Marie. Le compilateur a ainsi pris beaucoup de libertés avec ses sources, en intégrant des animaux absents de B (hirundo, milvus, herodius). Cette liberté d'intervention et de personnalisation de la matière des bestiaires est, nous l'avons vu, beaucoup plus prégnante dans les 11 premiers chapitres. À partir du lion, le compilateur est plus fidèle au Physiologus B, dans le choix des animaux, dans l'ordre des chapitres et dans la reprise textuelle de B, jusqu'à en donner des copies in extenso dans 10 des 12 derniers chapitres (si l'on ne compte pas les chapitres 30 et 31, qui sont des exempla).

\section{Deux notices particulières}

Pour illustrer les différences d'utilisation des sources par le compilateur du ms. 28 d'Avranches, voici deux notices, une au début du bestiaire (turtur), l'autre dans la seconde partie (hydrus/crocodillus). Les deux textes, confrontés aux chapitres équivalents dans le Physiologus B, sont donnés en annexe.

\section{Turtur (la tourterelle)}

La notice du turtur (annexe 2) n'est pas une copie directe de celle du Physiologus B, mais elle en partage plusieurs motifs: la fidélité et la chasteté de la tourterelle, la comparaison avec l'espérance dans la résurrection et dans le salut. Mais la rédaction du ms. 28 est fort différente du texte du Physiologus B: la notice commence par une citation biblique abrégée (également citée dans le Phys. B) : Vox turturis audita est [in terra nostra $]^{68}$. Puis le chapitre est structuré comme suit :

- similitudo/comparaison : la tourterelle est l'image de Marie et de la sainte église ;

- propriété : chasteté de la tourterelle après la mort de son mâle ;

- allégorie : comparaison avec la chasteté et la pureté du Christ et de la Vierge Marie - cette chasteté est opposée à la débauche des juifs et des démons ;

- moralisation : comparaison entre la chasteté de l'oiseau et celle nécessaire de l'homme, qui prendre exemple sur la tourterelle, pour ne pas sombrer dans la luxure, qui va l'emmener aux enfers.

Le compilateur abrège au maximum les propriétés de l'oiseau, telles qu'elles sont décrites dans le Physiologus B, mais ajoute la comparaison avec la Vierge Marie. Cette comparaison semble assez originale, dont je n'ai pas trouvé d'autre exemple probant, au contraire de celle avec l'ecclesia, extrêmement courante, non seulement dans le Physiologus, mais chez de nombreux Pères de l'Église et commentateurs bibliques (Augustin, Ambroise de Milan, Bède, Haimon d'Auxerre...) ou encore dans l'Aviarum d'Hugues de Fouilloy ${ }^{69}$. Le texte d'Avranches n'utilise pas les passages bibliques évoqués dans le Physiologus $B$ dans la partie finale moralisante, se montrant ainsi très indépendant de cette version pour ce chapitre. Il innove notamment par rapport à $\mathrm{B}$ en mentionnant la débauche des juifs et des démons.

\section{Hydrus (la mangouste)}

On ne trouve pour ce chapitre aucune rubrique, ni pied de mouche, ni signe capitulaire, qui signalerait le début du passage dans le ms. 28. La notice (voir annexe 3) a été copiée à la suite de celle sur l'onagre, alors que ces descriptions sont bien distinctes dans le 
Physiologus B. L'onagre n'est pas l'ennemi du crocodile dans les bestiaires. Dans le Physiologus B, le chapitre 21 évoque deux animaux : l'onagre et le singe (onager et simia). Dans le ms. 28, les notices sur l'onagre et le singe sont littéralement semblables à celles du Physiologus B. et l'on a peine à comprendre pourquoi le compilateur du bestiaire d'Avranches a séparé ces deux items et fait apparaître le singe avant l'onagre.

La notice est en fait inspirée par le chapitre sur l'hydrus, ennemi du crocodile dans le Physiologus $\mathrm{B}^{70}$. Le compilateur du ms. 28 ne donne pas le nom de l'hydrus. A-t-il effectué un saut de chapitre? Travaillait-il d'après un manuscrit mutilé ou lacunaire? La succession la succession onager-hydrus se trouve par exemple dans le manuscrit 638 de Rouen (f. 238), sans intertitre ni rubrique. Dans le codex de Rouen, le chapitre hydrus est introduit par la phrase Item est animal in Nilo flumine quod dicitur Ydris, phrase non reprise dans Avranches. Ici un saut du même au même ou un oubli de ligne est malgré tout peu envisageable, car la ligne du manuscrit de Rouen ne commence pas par Item... Mais le saut d'une phrase est tout à fait possible d'après un autre manuscrit.

La structure de la notice se présente comme suit:

- propriété : l'hydrus se laisse avaler par le crocodile, mais parvient à sortir de son corps en lui déchirant les entrailles;

- moralisation : le crocodile est l'image des enfers. Le Seigneur fait sortir les morts des enfers (image de la résurrection). Les citations bibliques à l'appui de cette moralisation viennent du chapitre « hydrus » de la version B.

Pour ce chapitre, l'influence du Physiologus B est indéniable, même s'il s'agit d'une copie de mauvaise qualité. Le texte présente des bizarreries syntaxiques, il manque parfois des mots, et quelques phrases sont difficiles à comprendre. En effet, l'auteur abrège souvent le texte dont il s'inspire (par exemple dans le passage où l'hydrus déchire les viscères du crocodile); il fait de même avec les citations bibliques qui sont fortement réduites par rapport à celles du Physiologus B.

\section{Conclusion}

Le compilateur du bestiaire fournit un travail de copie, mais aussi de réarrangement des chapitres et d'interprétation personnelle de la matière des bestiaires et des propriétés des animaux. Il ne respecte pas toujours l'ordre des chapitres du Physiologus B, en « réorganise » certains (cas de l'hydrus). Il mêle ainsi compilation de chapitres copiés assez fidèlement du Physiologus B et création de chapitres librement inspirés de $B$, ou de $Y$, réorganisés, avec des moralisations ou des interprétations allégoriques de son cru. Cette relative autonomie ne doit pas occulter l'utilisation massive du Physiologus $\mathrm{B}$, dont il ne subsiste aujourd'hui que très peu de témoins ${ }^{71}$. De plus, l'absence de certains animaux (panthère, éléphant, colombe, etc.) démontre que le copiste a probablement utilisé un manuscrit lacunaire, proche dans sa structure du manuscrit de référence de la version B, Bern, Bürgerbibliothek, Lat. 233, daté des VIII ${ }^{\mathrm{e}}$ $\mathrm{IX}^{\mathrm{e}}$ siècles), où ces mêmes animaux manquent ${ }^{72}$. Le bestiaire $\mathrm{d}^{\prime}$ Avranches, malgré son abord désordonné et improvisé, est intéressant car il atteste, au xiII siècle, une utilisation du Physiologus B qui, si elle n'est sans doute pas d'un apport essentiel quant à la tradition manuscrite de $B$, signale la circulation et l'utilisation d'une version ancienne de B ou d'un autre témoin de A, peut-être relié avec le manuscrit de Rouen, avec lequel, nous l'avons vu, il partage quelques points communs. Cette utilisation 
d'une version "archaïque» du Physiologus peut sembler surprenante, car les trois témoins aujourd'hui conservés de B sont antérieurs au $\mathrm{x}^{\mathrm{e}}$ siècle : le manuscrit de Bern, celui de Montecassino, Archivio dell'abbazia, 323, du IX ${ }^{e}$ siècle ${ }^{73}$; et celui d'Oxford, Bodleian Libr., Auct. T.2.23, du $\mathrm{IX}^{\mathrm{e}}$ siècle $\mathrm{e}^{74}$. Ceci est d'autant plus étonnant que de nouvelles versions du Physiologus vont avoir un succès et une diffusion importante à partir des $\mathrm{XI}^{\mathrm{e}}$ siècle et $\mathrm{XII}^{\mathrm{e}}$ siècle, comme en témoignent les plus de 130 manuscrits aujourd'hui conservés ${ }^{75}$.

Le bestiaire est complété par des exempla et des similitudines. Une notice sur les pierres ardentes directement reprise du Physiologus B, est insérée parmi les exempla, montrant ainsi une complémentarité entre des genres a priori différents: bestiaire, recueil d'exempla et de similitudines. Le bestiaire s'insère donc au sein d'un répertoire de thèmes moraux, directement réutilisables dans la prédication et la rédaction de sermons. L'ensemble Physiologus/exempla est lui-même contenu dans un ensemble codicologique regroupant des textes variés ayant tous un rapport direct avec la prédication : sermons, gloses, distinctiones, traités sur les vices et vertus, etc. Cette étude a ainsi confirmé la notice faite par Léopold Delisle, décrivant le ms. 28 d'Avranches comme étant un " recueil de matériaux à l'usage des prédicateurs ", dans lequel un bestiaire a tout à fait sa place ${ }^{76}$.

BIBLIOGRAPHIE

\section{Sources}

BERNARDINUS SENENSIS, Sancti Bernardini Senensis Opera omnia, tomus primus. 5 vol., Venezia, Andrea Poletti, 1745.

BESTIAIRE DE LA SECONDE FAMILlE : CLARK, Willene B. (éd.), A Medieval Book of Beasts. The SecondFamily Bestiary: Commentary, Art, Text and Translation, Woodbridge, Boydell, 2006.

GUILleRMuS ALVERnUS, Sermones, Guillelmi Alverni Parisiensis Opera Omnia, II, London, Robert Scot, 1674 .

HUGO DE FOLIETO, The Medieval Book of Birds: Hugh of Fouilloy's Aviarium, CLARK Willene B (éd.), Binghamton (N.Y.), Center for Medieval and Early Renaissance Studies, State University of New York, 1992 (Medieval and Renaissance texts and studies, 80).

JOHANNES DE SANCTO GEMINIANO, Liber de exemplis ac similitudinibus rerum, BRANT Sebastian (éd.), Basel, Johann Froben and Johann Petri de Langendorff, 1499. En ligne : http://tudigit.ulb.tudarmstadt.de/show/inc-ii-737.

MARTINUS BRACARENSIS, Martini episcopi Bracarensis Opera omnia, BARLow Claude W. (éd.), New Haven, Yale University press, 1950 (Papers and monographs of the American academy in Rome 12). 
MARTINUS BRACARENSIS, Sancti Martini Dumiensis episcopi Bracarensis Opuscula Septem, MIGNE JacquesPaul (éd.), Paris, Garnier-Migne, 1878 (Patrologia latina 72). En ligne : http://archive.org/details/ patrologiaecurs00migngoog.

PETRUS LOMBARDUS, P. Lombardi Opera omnia, MIGNE Jacques-Paul (éd.), Paris, Garnier-Migne, 1880 (Patrologia latina 191). En ligne : http://archive.org/details/patrologiaecurs122unkngoog. PHYSIOLOGUS A : CAHIER, Charles, et MARTIN Arthur (éd.), Mélanges d'archéologie, d'histoire et de littérature, II-III, Paris, Poussielgue-Rusand, 1851. En ligne : http://archive.org/details/ MelangesDArcheologieDHistoireEt3.

PHYSIOLOGUS B : CARMODY, Francis J. (éd.), Physiologus latinus. Éditions préliminaires versio B, Paris, Droz, 1939.

PHYSIOLOGUS Y : CARMODY, Francis J., (éd.), « Physiologus latinus versio Y », University of California Publications in Classical Philology 12, 1944, p 95-134. En ligne : https://archive.org/details/ universityofcali1213univuoft/page/n105 THOMAS CANTIMPRATENSIS, Liber de natura rerum, BOESE Helmut (éd.), Berlin - New York, W. de Gruyter, 1973.

VINCENTIUS BELLOVACENSIS. Speculum naturale. Douai, Baltazar Bellerus, 1624. En ligne : http:// sourcencyme.irht.cnrs.fr/encyclopedie/voir/133.

DICTA CHRYSOSTOMI : WILHELM, Friedrich (éd.), « Dicta Chrysostomi », in Denkmäler deutscher Prose des 11. und 12. B. Kommentar, 2/1, München, Callwey, 1916, p. 13-52. (Münchener Texte 8). En ligne : http://digital.ub.uni-duesseldorf.de/ihd/content/titleinfo/1763335.

SCHNEYER, Johannes Baptist (éd.), Repertorium der lateinischen Sermones des Mittelalters: für die Zeit von 1150-1350. 2, Autoren: E-H., Münster, Aschendorff, 1970 (Beiträge zur Geschichte der Philosophie und Theologie des Mittelalters 43).

ThEMA : Thesaurus Exemplorum Medii Aevi (ThEMA), BERLIOZ Jacques, POLO DE BEAULIEU Marie Anne, et collomB Pascal (éd.), Paris, GAHOM, 2006. En ligne : http://gahom.huma-num.fr/thema/.

THOMAS DE CHOBHAM, Summa de arte praedicandi, MORENZONI Franco (éd.), Turnhout, Brepols, 1988 (Corpus Christianorum, Continuatio medievalis 82).

\section{Études}

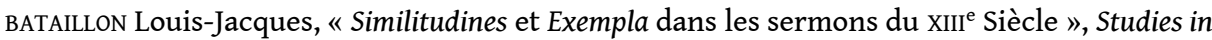
Church History. Subsidia 4, 1985, p. 191-205. En ligne : https://doi.org/10.1017/S0143045900003628. BAXTER Ron, Bestiaries and their users in the Middle Ages, Stroud - London, Sutton Publishing Courtauld Institute, 1998.

BISCHOFF Bernhard (éd.), Katalog der festländischen Handschriften des neunten Jahrhunderts. Teil II: Laon-Paderborn, Wiesbaden, Harrassowitz Verlag, 2004.

BUQUET Thierry, LECOUTEUX Stéphane, « Le catalogage des manuscrits du Mont Saint-Michel. L'exemple du Ms 222 d'Avranches ", Les Échos du Craham (carnet de recherche), 11 octobre 2017. En ligne : https://craham.hypotheses.org/1425.

DELISLE Léopold, Catalogue général des manuscrits des bibliothèques publiques des départements, publié sous les auspices du ministre de l'instruction publique, t. IV : Arras - Avranches Boulogne, Paris, Imprimerie nationale, 1872. 
JEUDY Colette, RIOU Yves-François, Les manuscrits classiques latins des bibliothèques publiques de France. Vol. 1, Paris, Éditions du CNRS, 1989.

KAY Sarah, Animal Skins and the Reading Self in Medieval Latin and French Bestiaries, Chicago London, The University of Chicago Press, 2017.

KAY Sarah, «'The English Bestiary', the Continental 'Physiologus', and the Intersections Between Them », Medium Ævum 85/ 1, 2016, p. 118-142. En ligne : https://doi.org/10.2307/26396473.

KUHRY Emmanuelle, «Dictionnaires, distinctions, recueils de propriétés en milieu cistercien : outils pour la prédication, sources pour l'étude de la nature », in TURCAN-VERKERK Anne-Marie, StUtZMann Dominique, falmagne Thomas et GANDIL Pierre (éd.), Les Cisterciens et la transmission des textes (XII ${ }^{e}-\mathrm{XVIII}^{e}$ siècles), Turnhout, Brepols, 2018 (Bibliothèque d'histoire culturelle du Moyen Âge 18), p. 285-337.

KUHRY Emmanuelle, « Les chapitres sur les poissons et créatures aquatiques dans la tradition manuscrite du Physiologus latinus et des bestiaires latins et leur réception dans quelques encyclopédies médiévales ", in GAUVIN Brigitte et LUCAS-AVENEL Marie-Agnès, Inter litteras \& scientias. Recueil d'études en hommage à Catherine Jacquemard, Caen, Presses universitaires de Caen, 2019 (coll. Miscellanea), p. 117-149.

LE MICHEL A., OUDIN P., Catalogus librorum manuscriptorum bibliothecae Sancti Michaelis in periculo maris, 1639. En ligne : https://gallica.bnf.fr/ark:/12148/btv1b10515937v/f231.image.

MC CuLLoch Florence, Medieval Latin and French Bestiaries, Chapel Hill, The University of North Carolina Press, 1962 (Studies in the Romance Languages and Literatures 33).

MORENZONI Franco, « Les animaux exemplaires dans les recueils de Distinctiones bibliques alphabétiques du XIII ${ }^{\mathrm{e}}$ siècle », in BERLIOZ Jacques et POLO DE BEAULIEU Marie-Anne (éd.), L'animal exemplaire au Moyen Âge ( $\left.V^{e}-X V^{e} s.\right)$, Rennes, Presses universitaires de Rennes, 1999 (Histoire), p. $171-190$.

MURATOVA Xenia, «Le bestiaire médiéval et la culture normande », in BOUET Pierre et DOSDAT Monique (éd.), Manuscrits et enluminures dans le monde normand ( $\mathrm{X}^{e}-\mathrm{XV} V^{e}$ siècles), Caen, Presses universitaires de Caen, 1999, p. 151-166.

PELLICER André, Natura : étude sémantique et historique du mot latin, Paris, Presses universitaires de France, 1966 (Publications de la Faculté des lettres et sciences humaines de l'Université de Montpellier 27).

\section{ANNEXES}

\section{Annexe 1 : Chapitres du bestiaire dans leur relation avec les versions du Physiologus latin}

\begin{tabular}{|l|l|l|l|l|l|}
\hline No & Chapitre & $\begin{array}{l}\text { Ordre } \\
\text { Phys. }\end{array}$ & $\begin{array}{l}\text { Marqueur } \\
\text { citation }\end{array}$ & $\begin{array}{l}\text { Marqueur } \\
\text { citation } \\
\text { Phys. dans }\end{array}$ & Rapports avec Phys. B \\
& B & Bhys. & \\
\hline
\end{tabular}




\begin{tabular}{|c|c|c|c|c|c|}
\hline 1 & Unicornis & 16 & Non & Oui & $\begin{array}{l}\text { Notice plus courte que B; ne } \\
\text { reprend pas les questions } \\
\text { étymologiques. Raccourcit la } \\
\text { description de la corne. Ne reprend } \\
\text { pas toutes les interprétations et les } \\
\text { citations bibliques de B. Rapport } \\
\text { lointain avec B. }\end{array}$ \\
\hline 2 & Turtur & 28 & Non & Oui & Rapport lointain avec B. \\
\hline 3 & Hirundo & & Non & - & $\begin{array}{l}\text { Absent de B. Lointain rapport avec } \\
\text { Y, notamment dans la citation } \\
\text { d'Ysaïe. Moralisation originale. }\end{array}$ \\
\hline 4 & Milvus & & Non & - & $\begin{array}{l}\text { Absent de B et } Y \text { (présent dans } \\
\text { certains manuscrits des } 2 \mathrm{e}, 3 \mathrm{e} \text { et } 4 \mathrm{e} \\
\text { familles). }\end{array}$ \\
\hline 5 & Cervus & 29 & Non & Oui & $\begin{array}{l}\text { Des points communs avec } \mathrm{Y} \text {; } \\
\text { moralisation originale ne } \\
\text { dépendant ni de } \mathrm{B} \text {, ni de } \mathrm{Y} \text {. }\end{array}$ \\
\hline 6 & Erinacius & 13 & Non & Oui & $\begin{array}{l}\text { Reprise de l'histoire des raisins; } \\
\text { moralisation originale ne } \\
\text { dépendant pas de B. }\end{array}$ \\
\hline 7 & Vulpis & 15 & Non & Oui & $\begin{array}{l}\text { Moralisation originale ne } \\
\text { dépendant pas de B. }\end{array}$ \\
\hline 8 & Caper & 20 & Non & - & $\begin{array}{l}\text { Reprise très résumée de la vue } \\
\text { acérée de la chèvre ; reprise } \\
\text { résumée de la moralisation sur les } \\
\text { prophètes. }\end{array}$ \\
\hline 9 & Herodius / Fulica & & Non & Non & $\begin{array}{l}\text { Les propriétés sont reprises de la } \\
\text { version } Y \text {, sans moralisation. }\end{array}$ \\
\hline 10 & Perdix & 25 & Non & Oui & $\begin{array}{l}\text { Les propriétés ont un lointain } \\
\text { rapport avec Y, mais pas la } \\
\text { moralisation, qui semble originale. }\end{array}$ \\
\hline 11 & Mustela & 26 & Non & Oui & $\begin{array}{l}\text { Les propriétés viennent de Y, mais } \\
\text { pas la moralisation, qui semble } \\
\text { originale. }\end{array}$ \\
\hline 12 & Leo & 1 & non & Oui & $\begin{array}{l}\text { Les } 3 \text { natures sont entremêlées avec } \\
\text { leurs moralisations. La notice, bien } \\
\text { qu'assez abrégée, est très fidèle à } \\
\text { celle du Phys. B, y compris dans les } \\
\text { moralisations. }\end{array}$ \\
\hline
\end{tabular}




\begin{tabular}{|c|c|c|c|c|c|}
\hline 13 & Adolaps & 2 & non & Non & $\begin{array}{l}\text { Propriétés reprises de Phys. B, mais } \\
\text { pas les moralisations. }\end{array}$ \\
\hline 14 & Pelicanus & 6 & non & Oui & $\begin{array}{l}\text { Propriétés résumées d'après B ; } \\
\text { reprise d'une moralisation sur la } \\
\text { crucifixion. }\end{array}$ \\
\hline 15 & Aquila & 8 & non & Oui & $\begin{array}{l}\text { Propriétés reprises de Phys. B, mais } \\
\text { moralisations plus longues ; le ms. } \\
\text { d'Avranches ne reprend pas } \\
\text { l'exemple sur les Juifs ; ni celui sur } \\
\text { le baptême. }\end{array}$ \\
\hline 16 & Upula & 10 & non & Oui & $\begin{array}{l}\text { Ne reprend pas les citations } \\
\text { bibliques initiales de B. Propriétés } \\
\text { abrégées; pas de reprise des } \\
\text { moralisations. }\end{array}$ \\
\hline 17 & Sora & 4 & non & Non & $\begin{array}{l}\text { Reprise de l'exemple nave vero } \\
\text { justorum ; reprise de la citation } \\
\text { biblique finale Qui perseverarit (Mt } \\
\text { 24:13) ; mais tout le reste est très } \\
\text { résumé d'après B. }\end{array}$ \\
\hline 18 & Caladrius & 5 & oui & Oui & $\begin{array}{l}\text { Notice très proche de } B \text {, reprise in } \\
\text { extenso ; ajout de } 2 \text { phrases finales. }\end{array}$ \\
\hline 19 & Nycticorax & 7 & non & Non & $\begin{array}{l}\text { Reprise de la propriété citée de } \mathrm{B} \\
\text { mais la moralisation est très } \\
\text { différente. }\end{array}$ \\
\hline 20 & Phenix & 9 & non & Oui & $\begin{array}{l}\text { Notice très proche de } \mathrm{B} \text {, reprise de } \\
\text { propriétés mais très légèrement } \\
\text { raccourcies. }\end{array}$ \\
\hline 21 & $\begin{array}{l}\text { Sirena et } \\
\text { Onocantaurus }\end{array}$ & 12 & oui & Oui & $\begin{array}{l}\text { Notice similaire à B, y compris la } \\
\text { moralisation. }\end{array}$ \\
\hline 22 & Simia & 31 & non & Non & $\begin{array}{l}\text { Notice similaire à B, y compris la } \\
\text { moralisation. }\end{array}$ \\
\hline 23 & Castor & 17 & oui & Oui & $\begin{array}{l}\text { Notice similaire à B, y compris la } \\
\text { moralisation. }\end{array}$ \\
\hline 24 & Hyena & 18 & oui & Oui & $\begin{array}{l}\text { Notice similaire à B, y compris la } \\
\text { moralisation. }\end{array}$ \\
\hline 25 & Caprea & 20 & oui & Oui & $\begin{array}{l}\text { Notice similaire à B, y compris la } \\
\text { moralisation. }\end{array}$ \\
\hline 26 & Onager & 21 & oui & Oui & $\begin{array}{l}\text { Notice similaire à B, y compris la } \\
\text { moralisation. }\end{array}$ \\
\hline
\end{tabular}




\begin{tabular}{|l|l|l|l|l|l|}
\hline 27 & [Crocodilus / hydrus $]$ & 19 & Oui & Oui & $\begin{array}{l}\text { Notice similaire à B, y compris la } \\
\text { moralisation. }\end{array}$ \\
\hline 28 & Stellio & 30 & Oui & Oui & $\begin{array}{l}\text { Notice similaire à B, y compris la } \\
\text { moralisation. }\end{array}$ \\
\hline 29 & Ibis & 14 & Non & Oui & $\begin{array}{l}\text { Notice proche de la première } \\
\text { moitié de celle de B }\end{array}$ \\
\hline 30 & Apicella & & Non & - & $\begin{array}{l}\text { Abeille absente des bestiaires. } \\
\text { Exemplum. }\end{array}$ \\
\hline 31 & {$[$ Aves $]$} & & Non & - & Exemplum. \\
\hline
\end{tabular}

\section{Annexe 2 : Chapitre sur la tourterelle (f. 179r, col. A)}

"Vox turturis audita est ${ }^{77}$. Turtur ista sanctam Ecclesiam vel sanctam Mariam significat. Si vero evenerit quod masculus ejus moriatur vel aliquo capiatur ingenio, alteri non commisebitur ${ }^{78}$ (sic), sed ipsum expectat et amoris ejus desiderio usque ad mortem servat castitatem. Audistis, fideles Christi, quanta castitas in tali avicula reperitur. Maria virgo vel sancta Ecclesia postquam vidit virum suum crucifixum et die tercia resurrexisse et ad celos ascendisse, contra eos nulle nequicie Judeorum vel demoniorum prevaluerunt, sed com justitia casti et puri usque in finem seculorum permanserunt. Ita et tu, homo, quare non servas castitatem uxori, et illa tibi ? Et si in die judicii illa castitas avicule a Domino laudatur, quid audituri sumus ab ipso propter luxurias et inquinamenta corporis nostri? Videamus ne luxuria nos demergat in puteum interitus ${ }^{79}$. Sed pura et immaculata castitas nos perducat ad consorcium angelorum, ubi Christus habitat in eternum. Amen.

«On a entendu le chant de la tourterelle ». La tourterelle signifie la Sainte Église ou Sainte Marie. S'il advient que le mâle de la tourterelle meure ou soit capturé par ruse, la femelle ne s'unira pas à un autre, mais elle attend son compagnon, et reste chaste jusqu'à la mort, regrettant son amour. Vous avez entendu, fidèles du Christ, quelle grande chasteté on trouve dans un aussi petit oiseau. [Telle est] la Vierge Marie, ou la Sainte Église, après avoir vu son époux ${ }^{80}$ crucifié et ressuscité au troisième jour, et monté au ciel. Toutes les débauches des Juifs et des démons n'ont pas réussi à triompher d'eux, et avec justice ils sont demeurés chastes et purs jusqu'à la fin des siècles. Toi, homme, pourquoi ne préserves-tu pas ainsi ta chasteté pour ta femme, et elle de même pour toi ? Et si au jour du Jugement dernier, la chasteté de ce tout petit oiseau est louée par le Seigneur, qu'entendrons-nous de sa part sur la luxure et les péchés de nos corps? Veillons à ce que la luxure ne nous fasse pas sombrer dans le puits de la mort. Mais puisse la chasteté pure et immaculée nous mener à la communauté des anges, où le Christ habite dans l'éternité. Amen.

\section{Chapitre du Physiologus B ${ }^{81}$}

Est volatile quod dicitur turtur; scriptum est de ea: Vox turturis audita est in terra. Physiologus de turture dicit valde virum suum diligere, et caste cum illo vivere, et ipsi soli fidem servare; ita ut si quando evenerit ut masculus eius aut ab accipitre aut ab aucupe capiatur, haec alteri 
masculo se non iungit, sed ipsum semper desiderat et ipsum per singula momenta sperat, et ipsius recordatione et desiderio usque ad mortem perseverat. Audite itaque, omnes animae fidelium, quanta castitas in modica avicula invenitur ; quicumque tamen personam turturis in vultu animae portatis, huius castitatem imitemini. Talis est enim sancta ecclesia, quae postquam vidit virum suum crucifixum, et die tertia resurrexisse et in caelos ascendisse, alio viro non coniungitur sed ipsum desiderat et ipsum sperat, et in illius amore et caritate usque ad mortem perseverat; dicente domino nostro Iesu Christo: "Qui perseveraverit usque in finem, hic salvus erit ${ }^{82}$. Similiter etiam et propheta David hortatur et dicit in psalmo: "Viriliter age, et confortetur cor tuum, et sustine dominum $"^{83}$.

\section{Annexe 3 : chapitre sur l'hydrus (f. 180r, col. A)}

Phisiologus dicit de e $0^{84}$ quod sit animal satis inimicum cocodrillo et hanc habere consuetudinem : cum viderit cocodrillum in flumine dormientem aperto ore, involvit se in limum luti, quo[d] facilius possit illabi in fauces ejus, et insilit in os ejus. Cocodrillus vero subito vivum transglutit eum. Ille vero dilanians eum vivus exit ab eo. Sic ergo mors et infernus figuram habent cocodilli (sic), qui inimicus est Domini ideo quod assumens terrenam carnem descendit ad infernum et disrumpens omnia ejus vasa ${ }^{85}$ eduxit eos qui ab eo tenebantur in morte, sicut testatur evuangelista: Monumenta aperta sunt et multa corpora surrexerunt ${ }^{86}$. Mortificavit sibi mortem quando a mortuis surrexit, sicut insultat illi propheta : "O mors, ero tua mors et morsus tuus ero, inferne $»^{87}$, et alibi : " Absorta est mors in victoria ; ubi est, mors, vita tua ? ${ }^{88}$

Le Physiologue dit de celui-ci [l'hydrus] qu'il est l'ennemi farouche du crocodile et qu'il a cette habitude : quand il voit le crocodile dormant la gueule ouverte sur le fleuve, il se roule dans la boue du limon afin de pouvoir s'infiltrer dans sa gorge plus facilement, et se lance dans sa gueule. Aussitôt, le crocodile l'avale vivant. Mais celui-ci en sort vivant en lui déchirant <les entrailles>. Ainsi, donc, la mort et l'enfer ont la forme du crocodile, qui est l'ennemi du Seigneur, parce que, prenant une chair terrestre, il est descendu en enfer, et brisant tous les vaisseaux de celui-ci, il en a fait sortir ceux qui étaient retenus là dans la mort, comme le dit l'Évangéliste : « les tombeaux ont été ouverts et beaucoup de corps ont ressuscité ». Il a tué la mort quand il a ressuscité d'entre les morts, et le Prophète prend à partie la mort en ces termes : « Ô mort, je serai ta mort et toi, Enfer, je serai ta morsure »; et ailleurs : « La mort a été engloutie dans la victoire; Mort, où est ta vie?».

\section{Chapitre du Physiologus B ${ }^{89}$}

Aliud animal est in Nilo flumine, quod dicitur hydrus. Physiologus dicit de eo quoniam satis hoc animal inimicum est crocodilo. Et hanc habet naturam et consuetudinem: cum viderit crocodilum in littore fluminis dormientem aperto ore, vadit et involvit se in limum luti, quod possit facilius illabi in faucibus eius, et veniens insilit in ore eius ; crocodilus igitur desubitatus, vivum transglutit eum ; ille autem dilanians omnis viscera eius exit vivus de visceribus crocodili iam mortui ac disruptis omnibus interraneis eius. Sic ergo mors et infernus figuram habent crocodili, qui inimicus est domini salvatoris nostri ; ideoque dominus noster Iesus Christus, assumens terrenam carnem nostram, descendit ad infernum et, disrumpens omnia viscera eius, eduxit omnes qui ab eo devorati detinebantur in morte; sicut testatur evangelista: Et monumenta aperta sunt, et resurrexerunt multa corpora sanctorum. Mortificavit igitur ipsam mortem; et ipse vivens resurrexit a mortuis et insulta illi per prophetam dicens: « 0 mors, ero 
mors tua; morsus tuus ero, inferne ». Et alibi : " Absorta est mors in victoria Christi; ubi est, mors, contritio tua; ubi est, mors, aculeus tuus?".

\section{NOTES}

1. Voir la notice en ligne du ms. 28 et consulter son fac-similé numérique sur le site de la BVMSM : $\quad$ https://www.unicaen.fr/bvmsm/ead.html? id=FR_UCBN_MSM_mss_av\&c=FR_UCBN_MSM_mss_av_Avranches_BM_28

2. MURATOVA, « Le bestiaire médiéval et la culture normande », $1999: 151$.

3. Je remercie Stéphane Lecouteux (Bibliothèque patrimoniale d'Avranches), Brigitte Gauvin (CRAHAM, Unicaen), Olivier Desbordes (Unicaen), notamment pour l'établissement du texte du bestiaire, Marjorie Burghart (CNRS, CIHAM). Je remercie également Emmanuelle Kuhry (IRHT) pour ses nombreux commentaires essentiels (relatifs à sa grande expertise sur la tradition manuscrite du Physiologus) sur une première version de ce texte, et Isabelle Draelants (IRHT) pour la relecture attentive de cet article et ses nombreuses suggestions.

4. LE MICHEL et OUDIN, Catalogus librorum manuscriptorum bibliothecae Sancti Michaelis in periculo maris, $1639: 104-114 \mathrm{v}$, sp.. 112 et 114.

5. JEUDY et RIOU, Les manuscrits classiques latins des bibliothèques publiques de France, vol. 1, 1989 : 211.

6. DELISLE, Catalogue général des manuscrits des bibliothèques publiques des départements, $1872: 442$.

7. On sait par exemple que le ms 222 d'Avranches a été acquis le 18 janvier 1390, auprès d'un maitre parisien, Simon Bedeau (Symon Bedellus). Voir BUQUET et LECOUTEUX, «Le catalogage des manuscrits du Mont Saint-Michel. L'exemple du Ms 222 d'Avranches », 2017.

8. Formule chiffrée : $1^{8}-7^{8}, 8^{4(-2)}, 9^{8}-11^{8}, 12^{8(-4)}, 13^{8}, 14^{10}-15^{10}, 16^{2}, 17^{8}-22^{8}, 23^{6}, 24^{2}, 25^{8(+1)}, 26^{8}, 27^{4}$, $28^{8}-30^{8}, 31^{4}, 32^{2}, 33^{4(-1)}$.

9. PIERRE LOMBARD, P. Lombardi Opera omnia, éd. MIGNe, Patrologia latina, 1880 : col. 97-104.

10. MARTINUS BRACARENSIS, Martini Episcopi Bracarensis Opera omnia, éd. BARLOW, 1950. Également édité par MIGNE, Patrologia latina, 1878 : col. 23C-26C.

11. JEUDY et RIOU 1989 , vol. $1: 211$.

12. DELISLE, $1872: 442-43$.

13. LE MICHEL et OUDIN, 1639 : f. 114 r.

14. Aux f. 147-147v, deux sermons sont de Geoffrey Babion (m. 1158), écolâtre d'Angers puis archevêque de Bordeaux. Je remercie Marjorie Burghart pour cette identification. Source signalée : SCHNEYER, Repertorium der lateinischen Sermones des Mittelalters, 1970 : 150-151 (sermons $\mathrm{T} 1$ et S10).

15. Inclus dans le catalogue du xIII ${ }^{e}$. de la bibliothèque de l'abbaye de Lyre, cité par MURATOVA, 1999 : 151 , note 3.

16. Cité par MURATOVA, $1999: 151$.

17. CLARK, A Medieval Book of Beasts. The Second-Family Bestiary 2006 : 119. Cette mention apparait, avec des variantes dans toutes les versions du Physiologus, y compris les plus anciennes.

18. Sur le rapport sémantique entre natura et proprietas dans l'Antiquité, voir PELICER, Natura : étude sémantique et historique du mot, 1966: 198-215. L'auteur signale que proprietas se trouve surtout dans les écrits philosophiques et techniques. Il y désigne le caractère propre, particulier, d'une chose (même emploi que le grec i̇iótn $:$ propriété, caractère propre). Selon Pelicer, natura est quant à lui un terme plus abstrait, lié au caractère originel, inné, instinctif, « naturel ».

19. THOMAS CANTIMPRATENSIS, Liber de natura rerum, éd. BOESE, $1973: 85$ (II, 4 et II, 6).

20. THOMAS CANTIMPRATENSIS, Liber de natura rerum, éd. BOESE, $1973: 3$.

21. PELICER, 1966: 198, note 3, explique qu'il faut bien faire la distinction entre proprietas (au singulier), dont l'emploi correspond le plus à natura, et les proprietates, caractères spécifiques. 
22. KUHRY, « Dictionnaires, distinctions, recueils de propriétés en milieu cistercien : outils pour la prédication, sources pour l'étude de la nature », $2018: 285-337$, surtout 297 et suivantes (chapitre sur la colombe).

23. KUHRY $2018: 332-333$.

24. THOMAS DE СHOBHAM, Summa de arte praedicandi, éd. MORENZONI, 1988 : prologue, p. 10: «tertium est ut sciat naturas animalium et etiam aliarum rerum, quia nichil est quod magis moveat corda auditorum quam proprietates animalium et aliarum rerum de quibus predicatur bene assignate, quia similitudines rerum quasi quedam novitates facilius et delectabilius movent animam ».

25. CARMODY, éd., Physiologus latinus. Éditions préliminaires versio B, 1939 : 13-14.

26. CLARK, $2006: 219$.

27. Voir note 24.

28. Berlioz, polo de BeAulieu, collomb (dir.), Thesaurus Exemplorum Medii Aevi (ThEMA), 2006-2018. http://thema.huma-num.fr/

29. ThEMA : http://thema.huma-num.fr/exempla/TE002927

30. ThEMA : http://thema.huma-num.fr/exempla/TE006520

31. ThEMA : http://thema.huma-num.fr/exempla/TE002953

32. ThEMA : http://thema.huma-num.fr/exempla/TE006559

33. ThEMA : http://thema.huma-num.fr/exempla/TE005690

34. ThEMA : http://thema.huma-num.fr/exempla/TE006531

35. GUillelmus alvernus, Sermones, Guillelmi Alverni Parisiensis Opera Omnia, II, 1674 : 402, Sermo 36 : In conversione sancti Pauli, sermo unicus.

36. JOHANNES DE SANCTO GEMINIANO, Liber de exemplis ac similitudinibus rerum, éd. Sebastian BRANT, 1499 : IX, 35, f. 312v.

37. BERNARDINUS SENENSIS, Sancti Bernardini Senensis Opera omnia, tomus primus, $1745: 52$.

38. BATAILLON, « Similitudines et Exempla dans les sermons du XIII ${ }^{\mathrm{e}}$ siècle », 1985 : 191-205.

39. MC CULLOCH, Medieval Latin and French Bestiaries, $1962: 25$.

40. KAY, Animal Skins and the Reading Self in Medieval Latin and French Bestiaries, 2017 : 84, note 62.

41. WILHELM, « Dicta Chrysostomi », 1916.

42. CARMODY, 1939.

43. Ce n'est plus vrai dans les versions ultérieures (H-B-Is, $H, 2^{\mathrm{e}}$ et $3^{\mathrm{e}}$ famille, Transitional) où les étymologies et le noyau du Physiologus sont entremêlés, donc plus difficiles à séparer. Je remercie E. Kuhry pour ces précisions.

44. CARMODY, éd., « Physiologus latinus versio Y », 1944 : 95-134, sp. 122-123.

45. Sicut leo et aquila immunda sunt. Sed leo omnium ferarum rex est, et aquila volatilium; et secundum hoc tantum Christo assimilantur, secundum rapacitatem - diabolicam - nequaquam (f. 179v, col. B)

46. Et sic multa sunt in creaturis duplicem sensum habencia vel intellectum, et in uno laudabilia et in alio vituperabilia (f. 179v, col. B).

47. CARMODY, $1944: 106$.

48. Et alia multa sunt in creaturis habencia duplicem intellectum: alia quidem sunt laudabilia, alia vituperabilia. (ms. Vat. Pal. lat. 1074, f. 3; voir https://digi.ub.uni-heidelberg.de/diglit/ bav_pal_lat_1074/0009/image).

49. Il manque également l'arbre Peredixion du Physiologus B, mais le compilateur du ms. d'Avranches n'a conservé que le règne animal, à l'exception des pierres ardentes placées à la fin du recueil de similitudines. Lacerta, présent dans l'édition de Carmody, est en fait un ajout de l'éditeur, sans doute pris du De bestiis ou des Dicta Chrysostomi. Je remercie E. Kuhry pour ces précisions relatives à l'édition de Carmody.

50. CARMODY, 1939 : 53-61. 
51. CARMODY, $1939: 53-54$.

52. CARMODY, 1939 : 53-54. Renseignement fourni par Emmanuelle Kuhry.

53. Je remercie E. Kuhry pour ces précisions.

54. CARMODY, 1939 : 40-52.

55. BAXTER, Bestiaries and their users in the Middle Ages, 1998 : 29-30.

56. Bruxelles, Bibl. royale, 10074, f. 140v-156v, $x^{e}$ s., édité dans CAHIER et MARTIN, 1851.

57. Bruxelles, Bibl. royale, 10066-77, f. 150v-151.

58. BAXTER, $1998: 29-30$.

59. Manuscrit signalé par E. Kuhry, que j'ai pu consulter à Rouen en 2018.

60. Le manuscrit d'Avranches donne fautivement milvius dans le texte.

61. Cet oiseau ne se trouve pas dans le texte de l'édition établie par CLARK, 2006.

62. HUGO DE FOLIETO, Aviarum, éd. CLARK, 1992 : 206-207.

63. Jérémie 8, 7 : Milvus in caelo cognovit tempus suum; turtur et hirundo et ciconia custodierunt tempus adventus sui.

64. THOMAS CANTIMPRATENSIS, Liber de natura rerum, éd. BOESE, 1973 : 214 : Queritur cur dominus in evangelio dixerit : milvus in celo cognovit tempus suum.

65. VINCENTIUS BELLOVACENSIS, Speculum naturale, XVI, 108, http://sourcencyme.irht.cnrs.fr/ encyclopedie/voir/133?citid=cit_id395592166368. Vincent cite le même passage sur Thomas de Cantimpré, mais en ajoutant comme marqueur de citation Glossa super Hieremiam: Aves sua tempora norunt, quando scilicet ad calida loca festinantes rigorem hyemis debeant declinare et a principio veris ad regiones solitas redire. Unde scriptum est. Milvus in celo cognovit tempus suum et cetera. Milvus non apparet nisi in estate, sicut nec turtur et hyrundo.

66. MORENZONI, «Les animaux exemplaires dans les recueils de Distinctiones bibliques alphabétiques du XIII ${ }^{\mathrm{e}}$ siècle ", 1999 : 179-180.

67. Le manuscrit Cambridge, Corpus Christi College, 22 présente un texte très modifié par rapport à son modèle $\mathrm{B}$, avec notamment l'ajout d'un chapitre, lacerta, qui ne se trouve jamais dans la famille B (ex. cité par BAXTER 1998 : 30, note 3).

68. Ct 2, 12 .

69. HUGO DE FOLIETO, Aviarum, éd. CLARK, 1992 : 154-155.

70. Sur le crocodile et l'hydrus dans les différentes familles de bestiaires, voir KUHRY «Les chapitres sur les poissons et créatures aquatiques dans la tradition manuscrite du Physiologus latinus et des bestiaires latins et leur réception dans quelques encyclopédies médiévales ", 2019 : 120-123.

71. BAXTER, $1998: 29$.

72. BISCHOFF, Katalog der festländischen Handschriften des neunten Jahrhunderts. Teil II: Laon-Paderborn, 2004 : 195-196.

73. BISCHOFF, $2004: 195-196$.

74. Il faut ajouter à cette liste le ms. Paris, Bnf, Latin 2825 (France du Nord, fin du $\mathrm{IX}^{\mathrm{e}} \mathrm{s}$.), qui donne, aux f. 49-56, une version très proche, avec quelques variantes, du Physiologus B. proximité qu'il faudrait étudier plus en détail (Cf. https://archivesetmanuscrits.bnf.fr/ark:/ 12148/cc60613q/cd0e935). Ce manuscrit a été récemment catalogué et mis en ligne (juillet 2017) dans le cadre du programme « France-Angleterre, 700-1200 » de la Fondation Polonsky.

75. Pour une mise au point récente de la diffusion des différentes versions des bestiaires, et une estimation du nombre conservé de témoins par famille voir KAY, “'The English Bestiary', the continental 'Physiologus', and the intersections between them », $2016: 118-142$, sp. 122-125.

76. Dans le catalogue médiéval de la bibliothèque de la Sorbonne (1341), on trouve la mention suivante d'un bestiaire utile à la prédication : Libellus qui bestiarum de naturis animalium et avium et aliarum rerum quarundum que valet ad predicandum. 'Leo fortissimus etc. (d'après CLARK, 2006 : 95).

77. Cf. Ct 2, $12:[. .$.$] vox turturis audita est in terra nostra.$ 
78. = commiscebitur. Cf., p. ex., Lv 15, 22 : cum masculo non commisceberis.

79. Comparer Ps 54, $24:$ Tu vero Deus deduces eos in puteum interitus.

80. Le Christ pouvait être fréquemment qualifié d' "époux ».

81. CARMODY, 1939 : 49-50.

82. Mt. 10.22 (Et eritis odio omnibus propter nomen meum: qui autem perseveraverit usque in finem, hic salvus erit) et Mt. 24.13 (Et quoniam abundavit iniquitas, refrigescet caritas multorum: qui autem perseveraverit usque in finem, hic salvus erit).

83. Ps. 26.14 : Expecta Dominum, viriliter age et confortetur cor tuum, et sustine Dominum.

84. Eo ne peut renvoyer à l'onagre ; il s'agit ici de l'hydrus, dont la notice évoque la description du crocodile dans le Physiologus B.

85. Viscera dans le Phys. B.

86. Cf. Mt 27, 52 : Et monumenta aperta sunt, et multa corpora sanctorum qui dormierant surrexerunt.

87. Cf. Os 13, $14:[. .$.$] ero mors tua, o mors, ero morsus tuus, inferne.$

88. Cf. 1 Co 15, 54-55: [...] absorta est mors in victoria. Ubi est, mors, victoria tua?

89. CARMODY, $1939: 35-36$.

\section{RÉSUMÉS}

Le manuscrit Avranches 28 est le fruit de la reliure de deux volumes distincts au XVII ${ }^{\mathrm{e}}$. On y trouve de très nombreux brefs traités religieux : commentaires et gloses bibliques, distinctiones, traités sur les vices et vertus, sermons, etc. Parmi tous ces matériaux à l'usage de la prédication, se trouve dans la seconde partie du manuscrit, datable du $\mathrm{XIII}^{\mathrm{e}} \mathrm{s}$., un bestiaire intitulé De proprietatibus quorundam animalium (f. 179-180). Ce bestiaire est le seul texte relatif aux animaux conservé de la bibliothèque du Mont Saint-Michel et est resté inédit. Au bestiaire est adjoint un bref recueil d'exempla, mettant notamment en scène des animaux, intitulé Ecce similitudines multe de diversis (f. 180-180v). Bestiaire et recueil de similitudines semblent former un ensemble peu différencié, qui aurait eu un même usage pour le compilateur de ces textes. Le bestiaire est composé d'une trentaine de courts chapitres, dont dix sont des décalques exacts de la version $\mathrm{B}$ $\mathrm{du}$ Physiologus latin; les autres chapitres ont de nombreux points communs avec B ou Y, mais sont souvent résumés ou abrégés, et comprenant des moralisations originales par rapport au Physiologus. J'envisage alors l'hypothèse que l'auteur du bestiaire du manuscrit d'Avranches a travaillé à partir d'un témoin incomplet de B où, comme dans le manuscrit de Bern (Bürgerbibliothek, Lat. 233), éléphant et colombe sont absents et autruche (asida), panthère, aspidochelon, sont placés à la fin du texte. Le bestiaire d'Avranches est donc intéressant à double titre : c'est un témoin (partiel) supplémentaire et inédit du Physiologus B, mais c'est aussi une œuvre mixte, originale dans sa composition et dans certains de ses chapitres, qui témoigne de la réception et de l'utilisation de versions anciennes du Physiologus chez les prédicateurs du XIII ${ }^{\mathrm{e}}$ siècle.

Manuscript 28 of Avranches is the result of the binding together of two distinct codices in the seventeenth century. It consists of various short religious texts: commentaries and biblical glosses, distinctiones, treatises on vices and virtues, sermons, etc. Among this extensive textual material for preaching use, in the second part of the manuscript (dating from the $13^{\text {th }}$ century), we find a bestiary entitled De proprietatibus quorundam animalium (f. 179-180). This is the unique text on animals surviving from the library of the Mont Saint-Michel Abbey. A short collection 
of exempla (partly involving animals) is added to the bestiary, and is entitled Ecce similitudines multe de diversis (f. 180-180v). The bestiary and the collection of similitudines seem to form a set; they may have had the same use for the compiler. The bestiary consists of about 30 short chapters, ten of which are perfect copies of the B version of the Physiologus; other chapters can be sourced partly in B or Y, but are often summarized and contain original moralizations which differ from other versions of the Latin Physiologus. I am making the assumption that the author of the bestiary of Avranches may have worked from an incomplete witness to B where the elephant and dove were missing, and where ostrich (asida), panther and aspidochelon were found the end of the text of the B version, such as in the codex of Bern, Bürgerbibliothek, Lat. 233. The bestiary of Avranches is interesting in two ways: it is an additional (though partial) witness to Physiologus B but is also a mixed work, original in its composition and in some of its chapters, testifying to the reception and use of ancient versions of the Physiologus among $13^{\text {th }}$ century preachers.

\section{INDEX}

Mots-clés : Mont Saint-Michel, bestiaire, Physiologus, prédication, exempla, Avranches ms. 28

Keywords : Mont Saint-Michel, Bestiary, Physiologus, Exempla, Avranches ms. 28, Preaching

\section{AUTEUR}

\section{THIERRY BUQUET}

Thierry Buquet est ingénieur de recherche CNRS, rattaché au CRAHAM (Université de Caen, UMR 6273). Diplômé de l'EPHE, sous la direction de Michel Pastoureau, ses recherches portent sur l'histoire des animaux exotiques et des ménageries au Moyen Âge, l'histoire des savoirs zoologiques et des zoonymes. Il est co-responsable au CRAHAM du programme ICHTYA (savoirs encyclopédiques sur les animaux marins et les poissons), et collabore au catalogage des manuscrits du Mont Saint-Michel. Il a publié une vingtaine d'articles, co-dirigé les actes du colloque Animaux aquatiques et monstres des mers septentrionales (http:// anthropozoologica.com/53/fasc2) et prépare actuellement une monographie sur l'histoire de la girafe. http://www.unicaen.fr/craham/spip.php?article927 